\title{
Testing for cointegration using induced-order statistics
}

\author{
Alvaro Escribano • M. Teresa Santos • \\ Ana E. Sipols
}

Accepted: 30 May 2006 / Published online: 5 September 2007

(C) Springer-Verlag 2007

\begin{abstract}
In this paper we explore the usefulness of induced-order statistics in the characterization of integrated series and of cointegration relationships. We propose a non-parametric test statistic for testing the null hypothesis of two independent random walks against wide cointegrating alternatives including monotonic nonlinearities and certain types of level shifts in the cointegration relationship. We call our testing device the induced-order Kolmogorov-Smirnov cointegration test (KS), since it is constructed from the induced-order statistics of the series, and we derive its limiting distribution. This non-parametric statistic endows the test with a number of desirable properties: invariance to monotonic transformations of the series, and robustness for the presence of important parameter shifts. By Monte Carlo simulations we analyze the small sample properties of this test. Our simulation results show the robustness of the induced order cointegration test against departures from linear and constant parameter models.
\end{abstract}

Keywords Unit root tests $\cdot$ Cointegration tests $\cdot$ Nonlinearity $\cdot$ Robustness $\cdot$ Induced order statistics · Engle and Granger test

This paper is an extension of the work of Aparicio and Granger (1995) and Aparicio and Escribano (1998).

A. Escribano $(\varangle)$

Departamento de Economía, Universidad Carlos III de Madrid, C./ Madrid, 126, 28903 Getafe (Madrid), Spain

e-mail: alvaroe@eco.uc3m.es

M. T. Santos

Department of Statistics, Universidad de Salamanca, Salamanca, Spain

\section{A. E. Sipols}

Department of Statistics, Universidad Rey Juan Carlos de Madrid, Madrid, Spain 


\section{Introduction}

Stochastic processes exhibiting cointegration will have similar long waves or similar long run behavior in their sample paths. Granger (1981) introduced the concept of cointegration, but it was not until Engle and Granger (EG) (1987) and Johansen $(1988,1991)$ that this concept gained immense popularity among econometricians and applied economists. When economic variables are non-stationary, cointegration helps avoiding the problem of spurious regressions, see Granger and Newbold (1974) and Phillips (1987). By now it is clear how to deal with integrated and cointegrated data in a linear context, see for example (Watson 1994; Johansen 1995) and Hendry (1995), but only a few recent papers have been dedicated to the simultaneous consideration of nonstationarity and nonlinearity, even though there is considerable consensus that these are important characteristics of many macroeconomic and financial economic relationships. Why has so little attention been devoted to this topic? The answer is clear; it is difficult to work with nonlinear time series models within a stationary and ergodic framework, and even more difficult within a nonstationary context. An introduction to the state of the art in econometrics relating nonlinearity and nonstationarity within a time series context can be found in Granger and Teräsvirta (1993), Granger (1995), Park and Phillips (2001), Bec and Rahbek (2004), Saikkonen (2005) and Escanciano and Escribano (2007). Granger (1995) discussed the concepts of longrange dependence in mean and extended memory that generalize the linear concept of integration, I(1), to a nonlinear framework. The main disadvantage of such definitions is that they have no Laws of Large Numbers (LLN), or Functional Central Limit Theorems (FCLT) associated with them, and it is therefore difficult to obtain estimation and inference results. On the other hand, there are interesting empirical macroeconomic applications in which nonlinearity has been found in a nonstationary context and, therefore, there is a need for those results to be justified econometrically.

Underlying the idea of cointegration is that of an equilibrium relationship (i.e., one that on average holds) between two cointegrated variables, $x_{t}, y_{t}$. A strict equilibrium exists when for some $\alpha \neq 0$, one has $y_{t}=\alpha x_{t}$. This unrealistic situation is replaced, in practice, by that of (linear) cointegration, in which the equilibrium error $z_{t}=y_{t}-\alpha x_{t}$ is a stationary time series. The concept of cointegration is linear since it is based on linear concepts of integration of order $d, I(d)$, see Marmol et al. (2002) for an alternative definition of cointegration using instrumental variables estimators. The usual concept of $I(d)$ is based on linear measures of dependence. Nonlinear measures of dependence, based on near epoch dependence, $\alpha$ - mixing or mutual information can be used to define a nonlinear concept of $I(d)$ and therefore nonlinear cointegration, see Aparicio and Escribano (1999), Escribano and Mira (2002) and Escribano et al. (2006). Nonlinear error correction means that the adjustment process towards the equilibrium is nonlinear and nonlinear cointegration refers to a nonlinear cointegration relationship. Furthermore, it should be pointed out explicitly that the same applies to the terms nonlinear error correction model and nonlinear cointegration model.

The relationship between cointegration and error correction model has been well characterized in a linear context (Granger's representation theorem), but its extension to the nonlinear context remains a challenge. Few extensions of the linear framework have been performed in the context of nonlinear error correction (NEC), see (Escribano 
1986, 1987b, 2004; Escribano and Mira 2002; Bec and Rahbek 2004) and Saikkonen (2005). Saikkonen (2005) also presents a Grangers's representation theorem for general nonlinear error correction models. Furthermore, the applied transformation of nonlinear vector equilibrium correction models into nonlinear vector autorregresive models may open the way for further theoretical work.

Under linear cointegration with nonlinear error correction adjustments the cointegrating errors are nonlinear. Several authors have analyzed those cases: (a) cubic polynomials, Escribano (1986, 2004), (b) rational polynomials, Escribano (2004), (c) threshold cointegration, Balke and Fomby (1997) (d) smooth transition regression models, Granger and Teräsvirta (1993) and general nonlinear autoregressive, Saikkonen (2005). Several nonlinear cointegration models have been discussed in the literature: (a) smooth transition cointegration functions, Choi and Saikkonen (2004), other parametric nonlinear cointegration functions, Aparicio et al. (2006a,b), nonparametric cointegration, Granger and Hallman (1991) and Aparicio and Escribano (1999). General nonparametric tests have the advantage of being valid under very general conditions and the disadvantage of not providing guidelines for particular parametric modeling. In the empirical application of this paper we will consider a simultaneous case of nonlinear cointegration and nonlinear error correction, see Sect. 4.

Although most cointegration studies rest on the assumption of a linear relationship between the variables, the possibility that these variables depend on each other through nonlinear relationships has opened up many questions for research. Since the concept of cointegration is inherently linear, some attempts have focused on extending standard definitions and on understanding how standard cointegration tests are affected by the presence of neglected nonlinearity, see Aparicio et al. (2006a). Hallman (1990a,b) and Granger and Hallman (1991) proposed the cointegrating nonlinear attractor concept for a pair of univariate integrated time series $x_{t}, y_{t} \sim I(d)$ by requiring the existence of nonlinear measurable functions $f(\cdot), g(\cdot)$ such that $f\left(x_{t}\right)$ and $g\left(y_{t}\right)$ are both $I(d), d>0$, and $s_{t}=f\left(x_{t}\right)-g\left(y_{t}\right)$ is $\sim I\left(d^{\prime}\right)$, with $d^{\prime}<d$.

Figure 1 illustrates the case of a cointegrating nonlinear attractor obtained by simulating a nonlinearly related pair of random walks with i.i.d. Gaussian errors. Aparicio and Granger (1995), Aparicio and Escribano (1998), Aparicio et al. (2006a,b), proposed the use of first differences of ranges and induced-order statistics to characterize cointegrating relationships. In this paper we analyze the properties of a new nonparametric test based on induced order statistics that is robust to monotonic nonlinear transformations and structural changes. In this paper we derive its asymptotic distribution and show, by Monte Carlo simulations, that the Engle and Granger (1987) test (EG test) fails dramatically in the presence of nonlinearities and structural breaks. We show that the well-known lack of robustness that affects most of the available tests of non-cointegration, (EG test, Johansen 1991) does not affect our IOC test. The small sample properties of the IOC nonparametric test are analyzed by Monte Carlo simulations. In particular, we test the null hypothesis of non-cointegration against the alternative hypothesis of a nonlinear error correction (NEC) and/or nonlinear cointegration. Furthermore, we are able to show that the IOC test for non-cointegration is robust for nonlinearities and structural breaks. One important advantage of this IOC approach is that it does not require prior estimation of the unknown (perhaps nonlinear) cointegrating relationship. 


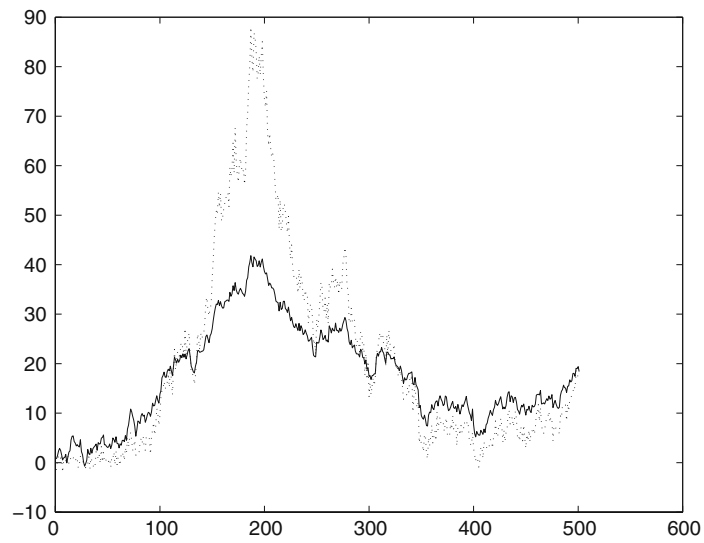

Fig. 1 A cointegrating nonlinear attractor obtained with a factorial model. The upper series was generated as $x_{t}=w_{t}+\epsilon_{t}$, while the lower one corresponds to $y_{t}=g\left(w_{t}\right)+\xi_{t}$, where $g(\cdot)$ represents a third-order polynomial of its argument random walk variable $w_{t}$, and $\epsilon_{t}$ are normally distributed

The structure of the paper is as follows. In Sect. 2 we introduce the IOC test, we derive its asymptotic distribution and study the consistency of the test. Section 3 analyses its power performances against different alternative hypotheses. In Sect. 4 we apply our IOC test to a nonlinear cointegration empirical example based on economic time series and compare the results with those obtained by means of standard noncointegration tests. Finally, after the concluding remarks of Sect. 5, the proofs of the main theoretical results are included in Appendix 1-3.

\section{Characterizing cointegration with induced-order statistics}

For the time series sample of size $\mathrm{n}$, say $x_{1}, \ldots, x_{n}$, the order statistics of $x_{t}$ are given by the sequence $x_{1, n} \leq \cdots \leq x_{n, n}$ obtained after a permutation of the indexes $1, \ldots, n$ such that $x_{i, n} \leq x_{i+j, n}, \forall j>0$. Related to order statistics are the so-called induced-order statistics (Bhattacharya 1984). The induced Y-order statistics based on the ordering of $x_{t}$ are defined as $\hat{y}_{i, n}=y_{j}$ if $x_{i, n}=x_{j}$; in general, notice that $\hat{y}_{i, n} \neq y_{i, n}$. Since for any order-preserving transformation such as monotonic nonlinear functions it follows that the order statistics are invariant, and this property was used by Hallman (1990a) to increase the robustness of the Dickey Fuller unit root test (DF) against monotonic nonlinear departures from the linear cointegration assumption. However, classical unit-root regression theory cannot be applied when the variables have discrete probability distributions, as in the case of rank variables. In fact, Breitung and Gouriéroux (1997) showed that the asymptotic null distribution is different in this case. Rank induced-order statistics should be useful for testing the existence of any sort of prominent low-frequency comovements, even in the more general cases of fractionally integrated time series and long-run relationships containing monotonic 
nonlinearities. However, they offer additional advantages, such as the possibility of constructing test statistics without nuisance parameters in their null distribution.

Let $\left\{P_{x}^{(n)}, P_{y}^{(n)}\right\}$ be sequences of $n \times n$ stochastic permutation matrices see Horn and Johnson (1990), defined as:

$$
\begin{aligned}
& P_{x}^{(n)} X=X_{(0)} \\
& P_{y}^{(n)} Y=Y_{(0)},
\end{aligned}
$$

where $Z=\left(z_{1}, \ldots, z_{n}\right)^{\prime}$ and $Z_{(0)}=\left(z_{1, n}, \ldots, z_{n, n}\right)^{\prime}, Z=X, Y$. The vector of induced Y-order statistics (induced by the ordering of X). $\hat{Y}=\left(\hat{y}_{1, n}, \ldots, \hat{y}_{n, n}\right)^{\prime}$ is obtained as

$$
\hat{Y}=P_{x}^{(n)} Y
$$

Now, since $P_{y}^{(n)}$ is a permutation matrix, it is invertible and $P^{-1}=P^{\prime}$, where $P^{\prime}$ denotes the transpose of $P$. Therefore, we can form the vector:

$$
\tilde{Y}=\left(P_{y}^{(n)}\right)^{-1} P_{x}^{(n)} Y
$$

Notice that for any order-preserving transformations, say $g(\cdot)$, we have

$$
\{g(X)\}_{(0)}=g\left(X_{(0)}\right)
$$

It follows that the order statistics of $y_{t}$ induced by the ordering of $s_{t}=g\left(x_{t}\right)$ will not change. That is, induced-order statistics are robust for monotonic nonlinearities in the DGPs of the series.

If $x_{t}$ and $y_{t}$ are cointegrated $\tilde{Y}$ should be close to $Y$, therefore $\hat{Y}$ and $Y_{(0)}$ should move together in the long run. However, if $x_{t}$ and $y_{t}$ are not cointegrated the behavior of $\tilde{Y}$ and $Y$ should be different since $X_{(0)}$ and $Y_{(0)}$ are very different. Therefore if $y_{t}$ and $x_{t}$ are cointegrated the permutations $P_{y}^{(n)}$ and $P_{x}^{(n)}$ should be identical in the long run. The intuition of this result can be seen in Fig. 2. In particular in Fig. 2a, we plot two independent random walks of $y_{t}$ and $x_{t}$, Fig. 2b, plots the corresponding induced order statistics $\widehat{Y}=P_{x}^{(n)} Y$ and $Y_{(0)}=P_{y}^{(n)} Y$. It is clear that there is no relationship between the two series of Fig. 2b. On the contrary, when the series are cointegrated, in Fig. 2c, the crossplot of both induced ordered series is around the $45^{\circ}$ line (slope equal to 1) as in Fig. 2d. Cointegrated series preserve the induced order in the long run.

In what follows we suggest testing the discrepancy between the induced ordered series based on the empirical distribution. We propose an alternative statistical measure of cointegration based on induced-order statistics to compare the orderings of the two time series $y_{t}$ and $\hat{y}_{t}$. The corresponding testing device will be referred to as the induced-order cointegration test (IOC). We shall now consider the KS statistic (Kolmogorov-Smirnov type statistics) defined below for testing the null hypothesis of two independent random walks

$$
K S=\sup _{j=1, n}\left|\hat{F}_{Y}^{(n)}\left(\hat{y}_{l_{s}(j), n}\right)-\hat{F}_{Y}^{(n)}\left(y_{j, n}\right)\right|,
$$



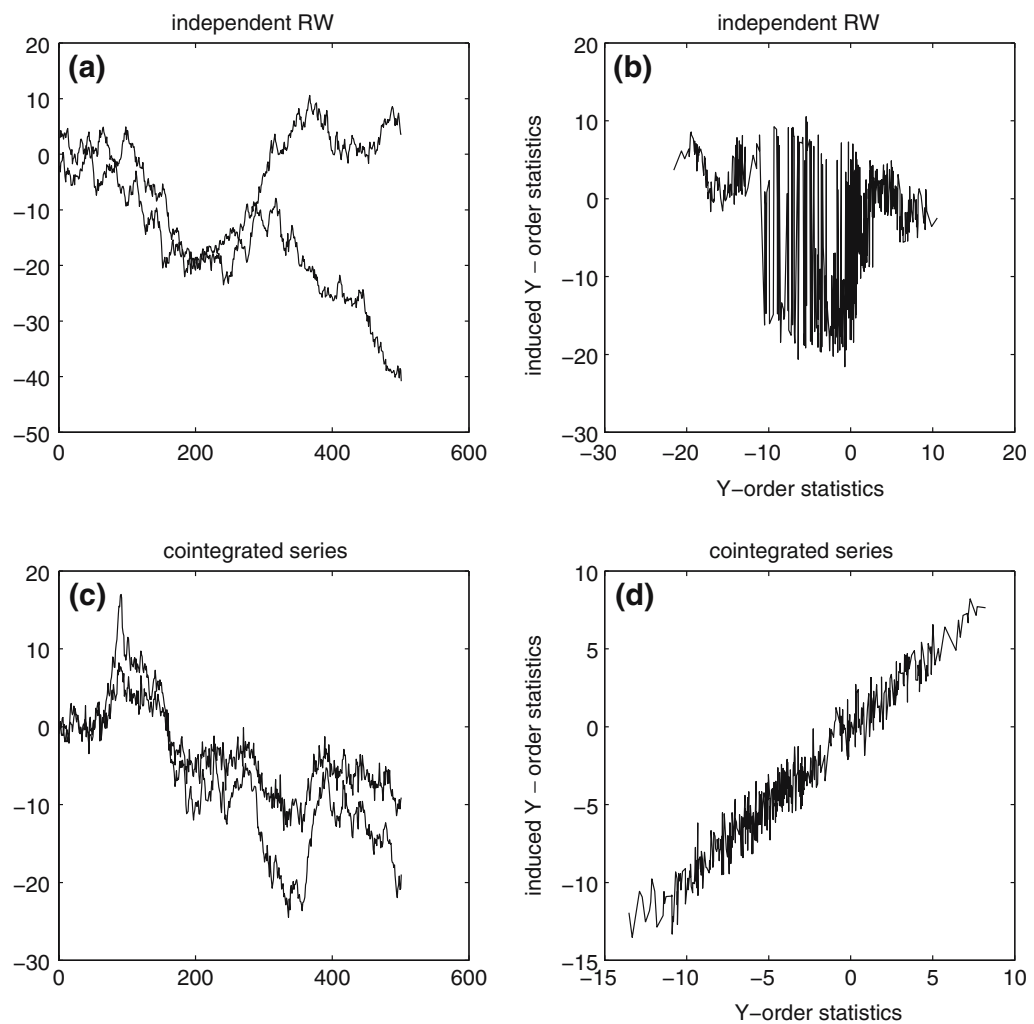

Fig. 2 a Two independent random walks, $\mathbf{b}$ the corresponding induced order statistics $\widehat{Y}=P_{X}^{(n)} Y$ in the $y$-axis and the order statistics $Y_{(0)}=P_{y}^{(n)} Y$ in the $x$-axis, $\mathbf{c}$ cointegrated series, and $\mathbf{d}$ the corresponding induced order statistics $\widehat{Y}=P_{x}^{(n)} Y$ in the $y$ axis and $Y_{(0)}=P_{y}^{(n)} Y$ in the $x$ axis

where $\hat{F}_{Y}^{(n)}(y)$ is the empirical distribution function obtained from a sample of length $n$ of $y_{t}$, that is, $\hat{F}_{Y}^{(n)}(y)=n^{-1} \sum_{t=1}^{n} 1\left(y_{t} \leq y\right)$, where $1(\cdot)$ denotes the indicator function; $s$ takes the sign of the cointegration parameter (which can be directly obtained from the scatter plot or simple regression of the two series), and $l_{s}(j)$ is equal to $j$ or to $n-j$, depending on whether $s=1$ or $s=-1$, respectively.

Smaller values of KS suggest that two independent random walks should be rejected, whereas large values indicate that two independent random walks hold. Therefore, we can consider the left tail of the distribution of K1 to discriminate between non-cointegrated and cointegrated series.

It is also easy to show that KS is robust against the order-preserving transformations of the variables, such as monotonic nonlinearities. If the actual cointegration relationship is nonlinear this test provides no guidelines for selection of particular parametric forms. 


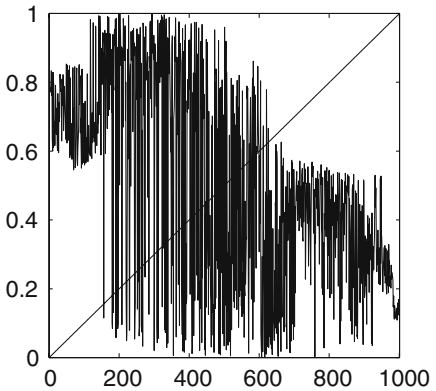

(a)

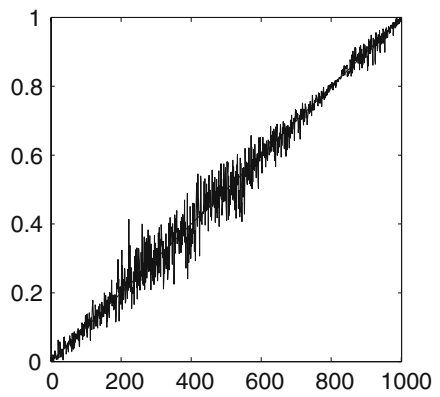

(c)

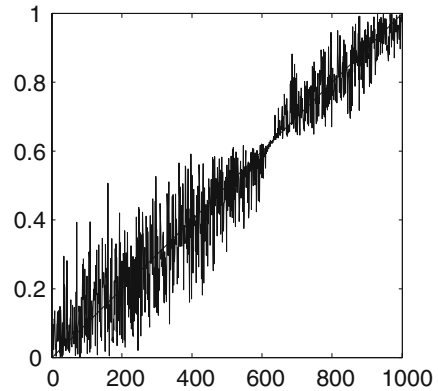

(b)

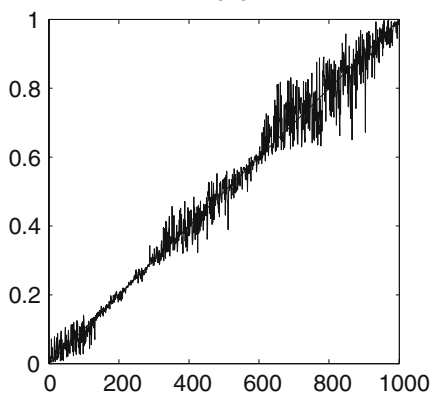

(d)

Fig. 3 Illustration of the proposed nonparametric cointegration measures for pairs of: a independent random walks, b series related through a quadratic attractor, $\mathbf{c}$ cointegrated series, and $\mathbf{d}$ linear cointegration with structural changes generated from Eq. 16

Our statistic is simply a particular distance measure between the sequences of order statistic $\hat{y}_{l_{s}(j), n}$ and $y_{j, n}$. Transforming these sequences with the empirical distribution function of $y_{t}$, say $\hat{F}_{Y}^{(n)}$, renders the statistic K1 unaffected by the variance of $y_{t}$, although it is still dependent on the signal-to-noise ratio in the relationship between $x_{t}$ and $y_{t}$.

In Fig. 3 we plot four different cases: (a) independent random walks, (b) quadratic polynomial attractor (nonlinear cointegration), (c) lineal cointegrated series and (d) linear cointegrated with structural breaks. In each case Fig. 3 shows the discrepancy between the sequences $\hat{F}_{Y}^{(n)}\left(y_{j, n}\right)$ and $\hat{F}_{Y}^{(n)}\left(\hat{y}_{l_{S}(j), n}\right)$. K1 is merely a measure of the variability of the sequence $\hat{F}_{Y}^{(n)}\left(\hat{y}_{l_{s}(j), n}\right)$ around the diagonal line, represented by the sequence $\hat{F}_{Y}^{(n)}\left(y_{j, n}\right)$. In the plots, the straight diagonal line corresponds to the sequence $\hat{F}_{Y}^{(n)}\left(y_{j, n}\right)$, while the superimposed series corresponds to $\hat{F}_{Y}^{(n)}\left(\hat{y}_{l_{s}(j), n}\right)$, where $s$ stands for the sign of the cointegration parameter.

We observe that when the series are cointegrated (linearly, nonlinearly and with structural breaks) the crossplot of the empirical distributions are centered around the $45^{\circ}$ line. Deviations are only transitory, while when the series are non 
cointegrated the discrepancies are permanent and most of the observations are off the $45^{\circ}$ line.

The KS statistic has been widely used both to test whether two random samples come from the same parent distribution and as a measure of distance between two probability distributions. Here we used it in a different sense, to obtain a discrepancy measure between the relative orders of $x_{t}$ and $y_{t}$ or, equivalently, of $y_{t}$ and the induced order series $\widehat{y}_{t}$.

We shall now derive the limit behavior of the null distribution of KS by using standard asymptotic theory for $I$ (1) processes. We shall first focus on deriving the asymptotic of the order and induced-order statistics for $I(1)$ time series. We shall invoke the continuous mapping theorem (CMT) to obtain the asymptotic of our test.

Theorem 2.1 Let $\hat{y}_{i, n}$ be the ith order statistic of $y_{t}$ induced by the ordering of $x_{t}$, where $x_{t} \sim I(1)$. Then,

$$
\frac{\hat{y}_{i, n}}{n^{1 / 2}} \Rightarrow \int_{0}^{1} W_{y}(r) \mathbf{1}\left(\int_{0}^{1} \mathbf{1}\left(W_{x}(s)<W_{x}(r)\right) d s=l\right) d r=\int_{0}^{1} W_{y}(r) G_{x}^{(l)}(r) d r
$$

where $G_{x}^{(l)}(r)$, represents a random process given by

$$
G_{x}^{(l)}(r)=\mathbf{1}\left(\int_{0}^{1} \mathbf{1}\left(W_{x}(s)<W_{x}(r)\right) d s=l\right),
$$

$W_{x}(\cdot)$ and $W_{y}(\cdot)$ are the corresponding Brownian motion process associated to $x_{t}$ and $y_{t}$, respectively, and “ $\Rightarrow$ ” denotes convergence in distribution as $n \rightarrow \infty$.

Proof See Appendix 1

Corollary 2.2 Under the hypothesis of independent random walks, $\frac{\hat{y}_{i, n}}{n^{1 / 2}}$ converges to zero in probability.

Proof See Appendix 2

Let $F_{y}^{(n)}$ represent the empirical distribution function of $y_{t}$, and let us recall that the rank of $x_{i}$ in the sample $\left\{x_{1}, \ldots, x_{n}\right\}$ is defined as $R_{x}\left(x_{i}\right)=\sum_{j=1}^{n} 1\left(x_{j}<x_{i}\right)$, such that $x_{i, n}=R_{x}^{-1}(i)=x_{\pi(i, n)}$, with $\pi$ (.) denoting a stochastic permutation applied to the indexes of a sample of size $n$ in order to have the observations ordered. We can then define the induced-order statistics of $y_{t}$ as $\hat{y}_{i, n}=y_{\pi(i, n)}$.

Theorem 2.3 Let $x_{t}=\sum_{i=1}^{n} \epsilon_{i}$, and $y_{t}=\sum_{i=1}^{n} \varepsilon_{i}$, where $\left\{\epsilon_{i}\right\}_{i \geq 1}$, and $\left\{\varepsilon_{i}\right\}_{i \geq 1}$ are continuous i.i.d. random variables with bounded and symmetric pdf, zero means, and finite variances. Then,

$$
K S \Longrightarrow \sup _{l \in(0, n)} \mid \int_{0}^{1} 1\left(W_{y}(s)<W_{y}(q(l)) d s-l \mid\right.
$$


Table 1 Critical values of the KS test statistics

\begin{tabular}{lllll}
\hline$v \backslash \mathrm{n}$ & 100 & 250 & 500 & 1,000 \\
\hline 0.01 & 0.3564 & 0.3665 & 0.3812 & 0.3726 \\
0.025 & 0.4059 & 0.4382 & 0.4591 & 0.4346 \\
0.05 & 0.4653 & 0.4980 & 0.4830 & 0.4915 \\
0.10 & 0.5248 & 0.5697 & 0.5649 & 0.5794 \\
0.90 & 0.9505 & 0.9681 & 0.9721 & 0.9780 \\
0.95 & 0.9703 & 0.9801 & 0.9860 & 0.9870 \\
\hline
\end{tabular}

(ii) If $x_{t}$ and $y_{t}$ are cointegrated, then we have $K S \underset{n \rightarrow \infty}{\longrightarrow} 0$ and here the IOC test is consistent against this sort of alternative.

Proof See Appendix 3

\section{Small-sample performance of the IOC test: Monte Carlo simulations}

In this section we provide simulation evidence in small samples such that the nonparametric test statistic KS is useful for testing our null hypothesis of two independent random walks. Let the data generation process (DGP) be the following independent random walks:

$$
\begin{aligned}
D G P: H_{0} \quad \Delta y_{t} & =w_{1 t} \\
\Delta x_{t} & =w_{2 t},
\end{aligned}
$$

where $w_{1 t}$ and $w_{2 t}$ are standard normal distributions and mutually independent.

In Table 1 we estimate the quintals of the empirical distribution of the $I O C$ test statistic, under $H_{0}$, for different significance values $v$ and different samples sizes, $n$, computed by using 50,000 replications of independent random walks with i.i.d. Gaussian errors. Figure 4 shows the corresponding empirical density of K1 estimated by kernel smoothing, using the Epanechnikov kernel for 1,000 replications for different sample sizes.

\subsection{Size and power of the IOC test: Monte Carlo simulation}

We shall analyze the power of the IOC test using the $5 \%$ left tail critical value, based on 10,000 replications of the Monte Carlo experiment. The DGP under the alternative hypothesis of cointegration is generated by a bivariate vector error correction model with weakly exogenous variables for the cointegrating parameter vector. Consider the following restricted VAR model for the $\left(y_{t}, x_{t}\right)$ vector, which is generated by

$$
\left[\begin{array}{l}
\Delta y_{t} \\
\Delta x_{t}
\end{array}\right]=\left[\begin{array}{l}
c \\
0
\end{array}\right]+\left[\begin{array}{l}
b \\
0
\end{array}\right](1,-\alpha)\left[\begin{array}{l}
y_{t-1} \\
x_{t-1}
\end{array}\right]+\left[\begin{array}{l}
w_{1 t} \\
w_{2 t}
\end{array}\right],
$$

where $w_{t}$ is standard Normal. 


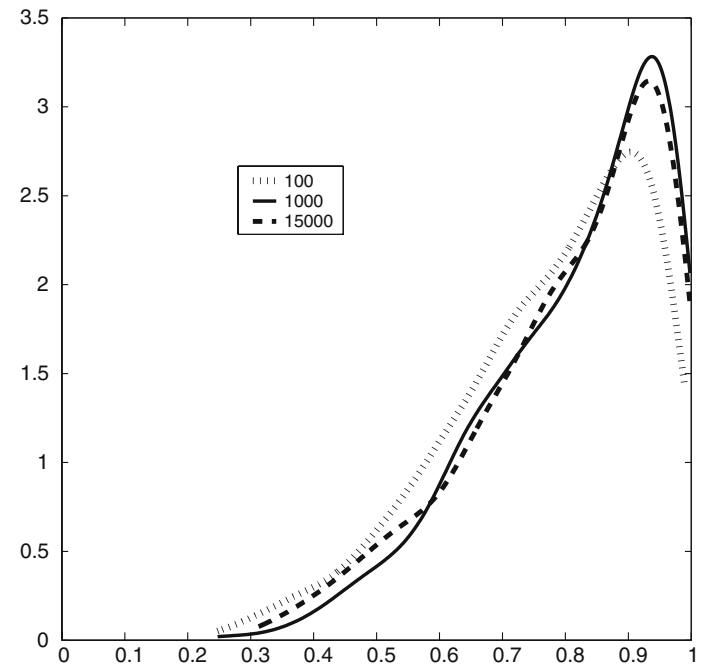

Fig. 4 Plot of the empirical density of KS under the null hypothesis for different sample sizes $n=100$, 1,000 and 15,000

Table 2 Empirical Size and Power of the KS and EG test based on $H_{1 a}$ at $5 \%$ significance level for different values of $b=0$ (no cointegration), $b=$ $-0.05,-0.5,-0.75$ (cointegration) and different sample sizes $n=100,250,500$

\begin{tabular}{|c|c|c|c|c|c|c|}
\hline \multirow[t]{2}{*}{$b \backslash n$} & \multicolumn{2}{|l|}{100} & \multicolumn{2}{|l|}{250} & \multicolumn{2}{|l|}{500} \\
\hline & $\mathrm{KS}$ & EG & $\mathrm{KS}$ & EG & $\mathrm{KS}$ & EG \\
\hline 0 & 0.05 & 0.051 & 0.051 & 0.052 & 0.05 & 0.05 \\
\hline-0.05 & 0.43 & 0.1450 & 0.51 & 0.59 & 0.6 & 0.98 \\
\hline-0.5 & 0.65 & 1 & 0.85 & 1 & 0.9 & 1 \\
\hline-0.75 & 0.7 & 1 & 0.9 & 1 & 0.91 & 1 \\
\hline
\end{tabular}

\subsubsection{Linear cointegration}

The alternative hypothesis is a standard linear error correction model (ECM):

$$
\begin{aligned}
D G P: H_{1 a} \quad \Delta y_{t} & =c+b\left(y_{t-1}-\alpha x_{t-1}\right)+w_{1 t} \\
\Delta x_{t} & =w_{2 t},
\end{aligned}
$$

where $\alpha=1$ and $c=0$. We shall study the power of different cointegration tests: IOC and EG test for different parameter values of the parameter $b$. We set $b=0$ (no cointegration), $b=-0.05,-0.5,-0.75$ (cointegration). This DGP follows the parameterization used by Kremers et al. (1992), and Arranz and Escribano (2001). The results are in Table 2 .

Since the EG test is based on estimating the true error correction model (DGP), the EG test for non-cointegration is more powerful than IOC. However, IOC is more powerful for small values of $b$; see, for example, $b=-0.05$ in Table 2 . 


\subsubsection{Nonlinear cointegration}

Escribano (1986, 2004) analyzed error correction models in nonlinear contexts where the cointegration relationship is linear or nonlinear and the equilibrium correction term could also be linear or nonlinear. Alternative representation theorems for nonlinear error correction (NEC) models based on general concepts of $\mathrm{I}(1)$ and $\mathrm{I}(0)$ were introduced by (Escribano 1986, 1987b; Escribano and Mira 2002; Bec and Rahbek 2004) and Saikkonen (2005). The main advantage of those definitions is that they have associated Laws of Large Numbers and Central Limit Theorems to derive the asymptotic properties of the estimators of the error correction models parameters. Escribano $(1986,2004)$ proposed a methodology for implementing parametric and nonparametric error correction models. Using the data bases of Friedman and Schwartz (1982) and Ericsson et al. (1997), extended until the year 2000, he implemented this methodology to estimate a nonlinear money demand in the UK from 1878 to 2000. In Sect. 4 we will apply our non-cointegration IOC test to this data set. Within the class of parametric models he discusses cubic polynomial (and rational polynomial) error correction models, see also Hendry and Ericsson (1991). Nonlinearities can eliminate most of the power of the usual non-cointegration test (EG test), as will be seen in the following Monte Carlo simulations.

\subsubsection{Power of IOC against a Nonlinear cointegrating relationship}

Let us now consider that the DGP under the alternative hypothesis is given by the following linear error correction model (ECM), with a nonlinear cointegration relationship.

$H_{1 b}$ : The alternative hypothesis is a linear ECM, but with nonlinear cointegration,

$$
\begin{aligned}
D G P: H_{1 b} \quad \Delta y_{t} & =c+b\left(y_{t-1}-g\left(x_{t-1}, \alpha\right)\right)+w_{1 t} \\
\Delta x_{t} & =w_{2 t},
\end{aligned}
$$

where $w_{1 t}$ and $w_{2 t}$ are standard normal distributions and mutually independent errors, with $\alpha=1$. Let the nonlinear cointegration relationship be given by the polynomial cointegration term $g\left(z_{t-1}, \alpha\right)=z_{t-1}^{j}$. Based on 10,000 replications of the Monte Carlo experiment, we now analyze the power of the I OC test at $5 \%$ significance level for different values of $b$, and we compare the results with those of the EG test, whose results are shown in Table 3.

As can be seen from Table 3, when the ECM is linear but with a polynomial cointegration function, the IOC test is much more powerful than EG in all cases. In particular, we observe that the highest power is obtained for $j=3$, followed by $j=4$ and $j=2$, respectively. The intuitive explanation given by Escribano (2004) is as follows: cubic polynomials are very flexible and can approximate different level shifts. Furthermore, the error correction term in this case can be equilibrium-correcting (stable nonlinear adjustment) and hence not a deviating error adjustment term, as may happen with the quadratic polynomial. We shall now consider other functional forms. Let the nonlinear 
Table 3 Empirical power of KS and EG based on $H_{1 b}$ with $g=z_{t-1}^{j}$ at $5 \%$ significance level for different values of $b$ and different sample sizes $n$

\begin{tabular}{|c|c|c|c|c|c|c|c|}
\hline & \multirow[t]{2}{*}{$j \backslash n$} & \multicolumn{2}{|l|}{100} & \multicolumn{2}{|l|}{250} & \multicolumn{2}{|l|}{500} \\
\hline & & KS & EG & KS & EG & KS & EG \\
\hline \multicolumn{8}{|l|}{$b=-0.05$} \\
\hline & 2 & 0.83 & 0.08 & 0.94 & 0.02 & 0.98 & 0.01 \\
\hline & 3 & 0.94 & 0.1 & 1 & 0.03 & 1 & 0.008 \\
\hline & 4 & 0.92 & 0.08 & 1 & 0.01 & 1 & 0.006 \\
\hline \multicolumn{8}{|l|}{$b=-0.5$} \\
\hline & 2 & 0.88 & 0.09 & 0.96 & 0.04 & 1 & 0.01 \\
\hline & 3 & 0.9 & 0.1 & 1 & 0.06 & 1 & 0.05 \\
\hline & 4 & 0.88 & 0.069 & 1 & 0.027 & 1 & 0.019 \\
\hline \multicolumn{8}{|l|}{$b=-0.75$} \\
\hline & 2 & 0.9 & 0.1 & 0.95 & 0.07 & 0.98 & 0.07 \\
\hline & 3 & 0.9 & 0.17 & 1 & 0.14 & 1 & 0.16 \\
\hline & 4 & 1 & 0.23 & 1 & 0.234 & 1 & 0.275 \\
\hline
\end{tabular}

Table 4 Empirical power of KS and EG based on $H_{1 b}$ with $g=\exp \left(z_{t-1} / 100\right)$ at $5 \%$ significance level for different values of $b$ and different sample sizes $n$

\begin{tabular}{lllllllll}
\hline$b \backslash n$ & 100 & & & 250 & & & 500 & \\
& KS & EG & & KS & EG & & KS & EG \\
\hline-0.05 & 0.43 & 0.05 & & 0.52 & 0.05 & & 0.57 & 0.05 \\
-0.5 & 0.65 & 0.07 & & 0.85 & 0.09 & & 0.9 & 0.1 \\
-0.75 & 0.7 & 0.1 & & 0.9 & 0.11 & & 1 & 0.15 \\
\hline
\end{tabular}

cointegrating function be $g\left(z_{t-1}, \alpha\right)=\exp \left(z_{t-1} / 100\right)$. The empirical powers of the IOC test and EG test for different values of $b$ are given in Table 4.

The power of the EG test is very low since this linear procedure misspecifies the estimation of the cointegrating vector by assuming that it is linear. In contrast, the power of the IOC test is very high. Similar results are obtained when the nonlinear cointegrating function is $g\left(z_{t-1}, \alpha\right)=\log \left(z_{t-1}+100\right)$.

\subsubsection{Linear cointegration with structural changes in the cointegrating vector}

There is a large body of literature concerning the effects of cointegration testing in the presence of structural changes, see Escribano (1987a). In what follows we wish to simulate a case based on the DGP of Arranz and Escribano (2001) to evaluate the power of IOC in this context when the break point is in the middle of the sample.

The alternative hypothesis is a linear error correction and cointegration model in the presence of a structural change in the cointegrating vector

$$
\begin{aligned}
D G P: H_{1 c} \Delta x_{t} & =w_{1 t} \\
\Delta y_{t} & =c+b\left[y_{t-1}-\left(c_{1} D_{1 t-1} x_{t-1}+\alpha x_{t-1}\right)\right]+w_{2 t},
\end{aligned}
$$


Table 5 Empirical power of KS and EG based on $H_{1 c}$ with $\alpha=1, c_{1}=2$ at $5 \%$ significance level for different values of $b$ and different sample sizes $n$

\begin{tabular}{lllllllll}
\hline$b \backslash n$ & 100 & & & 250 & & & 500 & \\
& KS & EG & & KS & EG & & KS & EG \\
\hline-0.05 & 0.7 & 0.15 & & 0.8 & 0.16 & & 0.95 & 0.17 \\
-0.5 & 0.81 & 0.23 & 1 & 0.25 & & 1 & 0.25 \\
-0.75 & 0.8 & 0.25 & 1 & 0.26 & & 1 & 0.29 \\
\hline
\end{tabular}

where $w_{1 t}, w_{2 t}$ are standard, and mutually independent normal errors, where $c_{1}$ measures the change in the cointegrating vector. We shall consider the values, $\alpha=1$, $c_{1}=2$ and, we shall understand that the structural break is created by the artificial dummy variable $D_{1 t}$, defined by:

$$
D_{1 t}= \begin{cases}1, & t \geq \frac{n}{2} \\ 0, & \text { otherwise. }\end{cases}
$$

Based on 10,000 replications of the Monte Carlo simulations, we obtain the empirical power of the IOC and EG tests, see Table 5.

Table 5 shows that the IOC is more powerful than the EG test in rejecting the null hypothesis of noncointegration in the presence of a structural break in the cointegrating vector. The power of IOC is really good when the parameter of the error correction adjustment, $b=-0.5$ or higher. For very low adjustment parameter values, $b=-0.05$, the empirical power of IOC is over $70 \%$ and much higher than EG-test, which is lower than $20 \%$.

\section{Empirical application}

\subsection{Analysis of the UK money demand (1878-2000)}

Escribano $(1986,2004)$ found a cointegration relationship between the logarithmic (logs) transformation of velocity of the circulation of money (V) and short run interest rates in nominal terms (RNA) from annual observations taken from 1878 to 2000 based on nonlinear error correction adjustments. Those error correction models consider several nonlinear parametric and nonparametric (smoothing splines) alternatives. Among the parametric nonlinear adjustments considered are cubic polynomials and rational polynomials of the cointegrating errors obtained from the nonlinear cointegration relationship between velocity of circulation of money and short run interest rates. Therefore, standard unit root test will not work well since they are not robust to these types of nonlinearities. This cointegration relationship is nonlinear (exponential) since the interest rate (RNA) is without logs while velocity of circulation of money is in $\operatorname{logs}(\mathrm{V})$, see Fig. 5.

When we test the null hypothesis of non-cointegration between V and RNA based on the residuals of the $E G$ test statistic we obtain a value of -1.7 , and therefore we cannot reject the null hypothesis of non-cointegration at the $5 \%$ significance level. This 

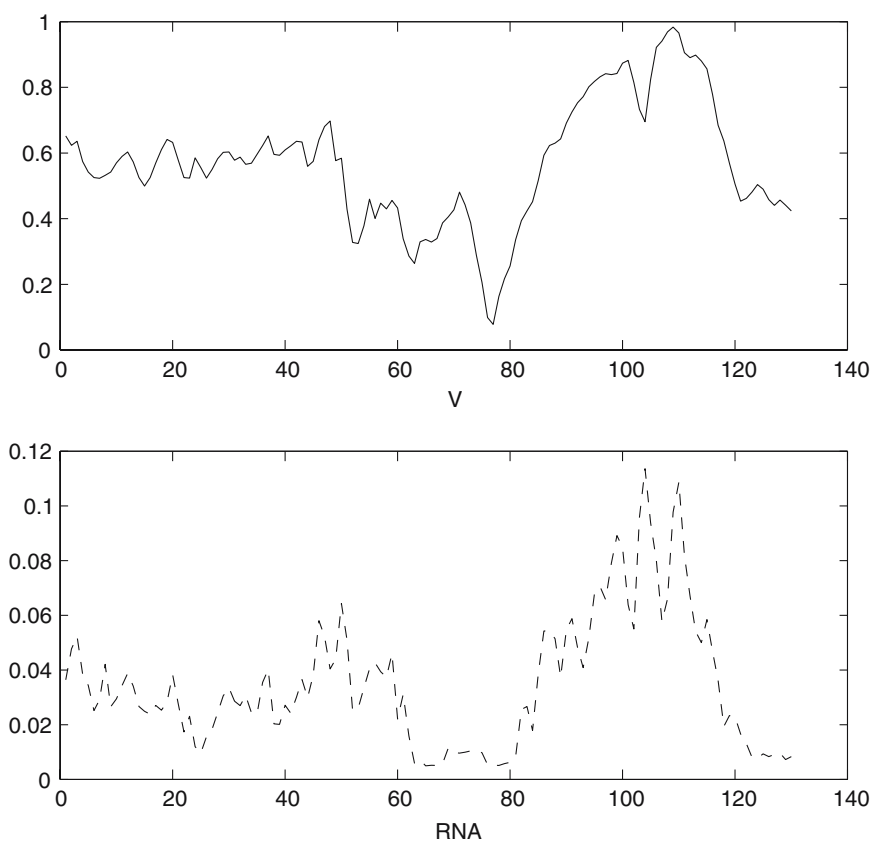

Fig. 5 Log of the velocity of the circulation of money (V) and short run interest rates in nominal terms (RNA)

result contradicts the ECM test carried out in the nonlinear error correction model, see Escribano (2004).

However, applying our IOC test statistic, we obtain $K S=0.2$, which is smaller than the $5 \%$ critical value, for $n=130$ (0.47). Therefore, using our induced order cointegration test IOC, we are able to reject the null hypothesis of non-cointegration.

\section{Conclusions}

Cointegration is an important property of many economic variables but in order to find convincing empirical evidence we usually need to extend the linear framework by allowing some time-varying parameter models or by considering nonlinear relationships. Standard cointegration tests, such as the EG test, are not robust for nonlinearities or for certain time-varying parameter models (level shifts, structural breaks, etc). In this paper, we have presented a model-free methodology that allows testing for the presence of cointegration in time series, and that is robust for the presence of monotonic nonlinearities and structural changes. These properties are very important because, on the one hand, standard cointegration tests are tailor-made to a specific parametric linear models for the individual series under the null hypothesis and, on 
the other, because in many applications one does not really know the transformation of the series that can linearize their relationship. For this, we propose an alternative testing device based on our induced-order test statistic (KS) from the series which has the advantage of not requiring prior estimation of the cointegration parameter, thereby leading to null distributions that are free of nuisance parameters. On the other hand it provides no clear guidelines for particular parametric modelling.

Extensions of our nonparametric approach to more than one cointegration vector, to larger multivariate systems or to linear trends are out of the scope of this paper and are left for future research.

Acknowledgments This research was partially supported by the project BEC2002-00279, SEJ200505485, SEJ2005-06454 and European project HPRN-CT-2002-00232. We are grateful to the Editor and to one referee for their comments. The authors are specially grateful to Felipe Aparicio who recently passed away. All errors are the entire responsability of the authors.

\section{Appendix 1}

Definition 6.1 A time series $x_{t}$ is said to be $I(0)$ if the process $X_{n}$ defined in the unit interval by

$$
X_{n}(\xi)=\sum_{t=1}^{[n \xi]}\left(\frac{x_{t}-E\left[x_{t}\right]}{\sigma_{x, n}}\right), \quad 0<\xi \leq 1
$$

where $\sigma_{x, n}^{2}=\operatorname{Var}\left[\sum_{t=1}^{n} x_{t}\right]$, denotes the long-run variance of $X_{n}$, converges weakly to a standard Brownian motion, $W_{x}$, as $n \rightarrow \infty$.

Let $x_{i, n}$ denote the $i$ th order statistic of an $I(1)$; that is, one that after the first differences becomes $I(0)$ following the definition by Davidson (1994) given before. We can write:

$$
x_{i, n}=\sum_{k=1}^{n} x_{k} \mathbf{1}\left(\sum_{i=1}^{n} 1\left(x_{j}<x_{k}\right)=i\right) .
$$

We can easily obtain

$$
\begin{aligned}
\frac{x_{i, n}}{n^{1 / 2}} & \Rightarrow \int_{0}^{1} W_{x}(r) \mathbf{1}\left(\int_{0}^{1} \mathbf{1}\left(W_{x}(s)<W_{x}(r)\right) d s=l\right) d r \\
& =\int_{0}^{1} W_{x}(r) G_{x}^{(l)}(r) d r .
\end{aligned}
$$

where " $\Rightarrow$ " denotes convergence in distribution as $n \rightarrow \infty$. Following Breitung and Gouriéroux (1997), $G_{x}^{(l)}(r)$ represents a random process given by 


$$
\begin{aligned}
G_{x}^{(l)}(r) & =\mathbf{1}\left(\int_{0}^{1} \mathbf{1}\left(W_{x}(s)<W_{x}(r)\right) d s=l\right) \\
& =\mathbf{1}\left(\int_{0}^{1} \mathbf{1}\left(W_{x}(r-s)>0\right) d s+\int_{r}^{1} \mathbf{1}\left(W_{x}(s-r)>0\right) d s=l\right) \\
& =\mathbf{1}\left(r A_{1}+(1-r) A_{2}=l\right),
\end{aligned}
$$

with $A_{1}, A_{2}$ denoting two independent random variables with an arcsin distribution. Note that $\int_{0}^{1} \mathbf{1}\left(W_{x}(s)<W_{x}(r)\right) d s$ represents the occupation time of the set $\left(-\infty, W_{x}(r)\right)$ by the Brownian motion, $W_{x}$, and $G_{x}^{(l)}(r)$ takes the value 1 whenever this occupation time equals $l \in(0,1)$ and 0 otherwise. Therefore, when $x_{t} \sim I(1)$ then $\frac{x_{i, n}}{n^{1 / 2}}$ converges weakly to a stochastic process indexed by $l$. In contrast, if $x_{t} \sim I(0)$ then it will converge to zero in probability.

Similarly, by allowing $\hat{y}_{i, n}$ to represent the $i$ th order statistic of $y_{t}$ induced by the ordering of $x_{t}$, we can write:

$$
\hat{y}_{i, n}=\sum_{k=1}^{n} y_{k} \mathbf{1}\left(\sum_{i=1}^{n} 1\left(x_{j}<x_{k}\right)=i\right) \text {, }
$$

and we obtain:

$$
\begin{aligned}
\frac{\hat{y}_{i, n}}{n^{1 / 2}} & \Rightarrow \int_{0}^{1} W_{y}(r) \mathbf{1}\left(\int_{0}^{1} \mathbf{1}\left(W_{x}(s)<W_{x}(r)\right) d s=l\right) d r \\
& =\int_{0}^{1} W_{y}(r) G_{x}^{(l)}(r) d r,
\end{aligned}
$$

with $l$ and $G_{x}^{(l)}(r)$ given as before. Note that if $x_{t}$ and $y_{t}$ are independent then the limiting process in the previous equation vanishes to zero in probability. We can even work out the nature of these limiting processes a little more. Let us focus on the behavior of $\int_{0}^{1} W_{y}(r) G_{x}^{(l)}(r) d r$ under cointegration, and let $S_{x}^{(l)}$ be the stochastic set given by

$$
S_{x}^{(l)}=\left\{r \in(0,1): r A_{1}+(1-r) A_{2}=l\right\},
$$

which for each possible value of the pair of random variables $\left(A_{1}, A_{2}\right)$, say $\left(a_{1}, a_{2}\right)$, will be formed by the single point:

$$
r_{l}=\frac{l-a_{2}}{a_{1}-a_{2}}
$$


In fact, $S_{x}^{(l)}$ is a random variable, and we may write:

$$
\int_{0}^{1} W_{y}(r) G_{x}^{(l)}(r) d r=\int_{S_{x}^{(l)}} W_{y}(r) d r
$$

which gives $\int_{S_{x}^{(l)}} W_{y}(r) d r=W_{y}\left(\frac{l-A_{1}}{A_{1}-A_{2}}\right)$ if $\frac{l-A_{1}}{A_{1}-A_{2}}$ belongs to the interval $(0,1)$ and 0 otherwise. Thus, we obtain a limiting non-Gaussian doubly stochastic process with positive probability mass at zero. Indeed, the Brownian motion process, $W_{y}$, is indexed by the process on the unit interval $q_{x}(l)=\frac{l-A_{1}}{A_{1}-A_{2}}$ indexed by $l$.

\section{Appendix 2}

Let us compute the mean and the variance of $\int_{S_{x}^{(l)}} W_{y}(r) d r$. First, for the mean, let us notice that

$$
\begin{aligned}
E\left[\int_{0}^{1} W_{y}(r) G_{x}^{(l)}(r) d r\right] & =E_{M}\left[E\left(W_{y}\left(\frac{l-A_{1}}{A_{1}-A_{2}}\right) \backslash A_{1}=a_{1}, A_{2}=a_{2}\right)\right] \\
& =\iint_{M} V_{l}\left(a_{1}, a_{2}\right) f_{A_{1}}\left(a_{1}\right) f_{A_{2}}\left(a_{2}\right) d a_{1} d a_{2},
\end{aligned}
$$

where $M$ represents the set given by those pairs of values $\left(a_{1}, a_{2}\right)$ such that either $a_{1}<l$ and $a_{2}<2 a_{1}-l$, or $a_{1}>l$ and $a_{2}>2 a_{1}-l$, and $f_{A}($.) stands for the probability density of the random variable $A$, and

$$
V_{l}\left(a_{1}, a_{2}\right)=E\left[W_{y}\left(\frac{l-A_{1}}{A_{1}-A_{2}}\right) \backslash A_{1}=a_{1}, A_{2}=a_{2}\right]
$$

However, it is clear that $V_{l}\left(a_{1}, a_{2}\right)=0$, such that $E\left[\int_{0}^{1} W_{y}(r) G_{x}^{(l)}(r) d r\right]=0$.

Regarding the variance of $\int_{S_{x}^{(l)}} W_{y}(r) d r$, from the previous result we have:

$$
\begin{aligned}
\operatorname{Var}\left[\int_{S_{x}^{(l)}} W_{y}(r) d r\right] & =E\left[\int_{0}^{1}\left(W_{y}(r) G_{x}^{(l)}(r) d r\right)^{2}\right] \\
& =E_{M}\left[E\left[W_{y}^{2}\left(\frac{l-A_{1}}{A_{1}-A_{2}}\right) \backslash A_{1}=a_{1}, A_{2}=a_{2}\right]\right] \\
& =\iint_{M} \frac{l-a_{1}}{a_{1}-a_{2}} f_{A_{1}}\left(a_{1}\right) f_{A_{2}}\left(a_{2}\right) d a_{1} d a_{2}
\end{aligned}
$$




$$
\begin{aligned}
= & \frac{4}{\pi^{2}} \int_{0}^{l} \int_{0}^{2 a_{1}-l} \frac{l-a_{1}}{a_{1}-a_{2}}\left(1-a_{1}^{2}\right)^{-1 / 2}\left(1-a_{2}^{2}\right)^{-1 / 2} \\
& +\frac{4}{\pi^{2}} \int_{l}^{1} \int_{2 a_{1}-l}^{1} \frac{l-a_{1}}{a_{1}-a_{2}}\left(1-a_{1}^{2}\right)^{-1 / 2}\left(1-a_{2}^{2}\right)^{-1 / 2} .
\end{aligned}
$$

Here, we have used the fact that $\operatorname{Var}\left[W_{y}(r)\right]=r$. Note that if $x_{t}$ and $y_{t}$ were independent we would obtain

$$
\begin{aligned}
\operatorname{Var}\left[\int_{S_{x}^{(l)}} W_{y}(r) d r\right] & =E\left[\left(\int_{0}^{1} W_{y}(r) G_{x}^{(l)}(r) d r\right)\left(\int_{0}^{1} W_{y}\left(r^{\prime}\right) G_{x}^{(l)}\left(r^{\prime}\right) d r^{\prime}\right)\right] \\
& =\int_{0}^{1} \int_{0}^{1} E\left[W_{y}(r) W_{y}\left(r^{\prime}\right)\right] E\left[G_{x}^{(l)}(r) G_{x}^{(l)}\left(r^{\prime}\right)\right] d r d r^{\prime} \\
& =\int_{0}^{1} \int_{0}^{1} \min \left(r, r^{\prime}\right) E\left[G_{x}^{(l)}(r) G_{x}^{(l)}\left(r^{\prime}\right)\right] d r d r^{\prime} .
\end{aligned}
$$

However,

$$
E\left[G_{x}^{(l)}(r) G_{x}^{(l)}\left(r^{\prime}\right)\right]=E\left[\mathbf{1}\left(r A_{1}+(1-r) A_{2}=l\right) \mathbf{1}\left(r^{\prime} A_{1}+\left(1-r^{\prime}\right) A_{2}=l\right)\right],
$$

which is equal to zero unless $r=r^{\prime}$, and hence:

$$
\operatorname{Var}\left[\int_{S_{x}^{(l)}} W_{y}(r) d r\right]=\int_{0}^{1} r E\left[G_{x}^{(l)}(r)\right]^{2} d r .
$$

Finally, we remark that

$$
\begin{aligned}
E\left[G_{x}^{(l)}(r)\right]^{2} & =P\left(G_{x}^{(l)}(r)=1\right) \\
& =P\left(\mathbf{1}\left(r A_{1}+(1-r) A_{2}=l\right)=1\right) \\
& =P\left(r A_{1}+(1-r) A_{2}=l\right) \\
& =0
\end{aligned}
$$


since $r A_{1}+(1-r) A_{2}$ is a continuous random variable for each $r$. Thus,

$$
\operatorname{Var}\left[\int_{S_{x}^{(l)}} W_{y}(r) d r\right]=0 .
$$

This result entails that under the hypothesis of independent random walks $\frac{\hat{y}_{i, n}}{n^{1 / 2}}$ must converge to zero in the quadratic mean, and therefore also in probability. However, when the series are cointegrated $\frac{\hat{y}_{i, n}}{n^{1 / 2}}$ will converge weakly to a double stochastic process obtained from a Brownian motion process.

\section{Appendix 3}

We have

$$
\begin{aligned}
F_{y}^{(n)}\left(\hat{y}_{i, n}\right) & =n^{-1} R_{y}\left(\hat{y}_{i, n}\right) \\
& =n^{-1} \pi^{*}(i, n),
\end{aligned}
$$

where $\pi^{*}(\cdot)$ denotes a different stochastic permutation of the indexes. Therefore:

$$
\begin{aligned}
K 1 & =\sup _{i=1, n}\left|F_{y}^{(n)}\left(\hat{y}_{i, n}\right)-F_{y}^{(n)}\left(y_{i, n}\right)\right| \\
& =n^{-1} \max _{1 \leq i \leq n}\left|\pi^{*}(i, n)-i\right| .
\end{aligned}
$$

Now, if the series $x_{t}$ and $y_{t}$ are cointegrated (either linearly or monotonically nonlinearly) we would expect $\pi^{*}(i, n)-i$ to remain close to zero, $\forall i$, in the range $1 \leq i \leq n$. Following Breitung and Gouriéroux (1997), we have:

$$
\begin{aligned}
n^{-1} R_{y}\left(\hat{y}_{i, n}\right) & =n^{-1} \sum_{t=1}^{n} 1\left(y_{t}<y_{\pi(i, n)}\right) \\
& =n^{-1} \sum_{t=1}^{n} 1\left(n^{-1 / 2} y_{t}<n^{-1 / 2} y_{\pi(i, n)}\right) \\
& =\sum_{t=1}^{n} 1\left(n^{-1 / 2} y_{\left[\frac{t}{n} n\right]}<n^{-1 / 2} y_{\left.\left[\frac{\pi(i, n)}{n} n\right]\right)}\left[\frac{t}{n}-\frac{t-1}{n}\right]\right. \\
& \Longrightarrow \int_{0}^{1} 1\left(W_{y}(s)<W_{y}(q(l)) d s,\right.
\end{aligned}
$$

where $q(l)=\frac{\pi(i, n)}{n}=n^{-1} \pi(n l, n)$ and $l=\frac{i}{n}$. Under cointegration $\hat{y}_{i, n}=y_{i, n}+\zeta_{i}$, where $\zeta_{i}$ is $I(0)$. It follows that $n^{-1} R_{y}\left(\hat{y}_{i, n}\right) \stackrel{p}{\longrightarrow} l$, such that $K S \stackrel{p}{\longrightarrow} 0$. In contrast, for non-cointegrated series, by the CMT 


$$
K S \Longrightarrow \sup _{l \in(0, n)} \mid \int_{0}^{1} 1\left(W_{y}(s)<W_{y}(q(l)) d s-l \mid\right.
$$

which represents a random variable taking strictly positive values with probability one.

\section{References}

Aparicio FM, Escribano A (1998) A nonlinear framework for testing cointegration using induced-order statistics. Working Paper 98-07, University Carlos III of Madrid

Aparicio FM, Escribano A (1999) Information theoretic analysis of serial correlation and cointegration. Stud Nonlinear Dyn Econom 3:119-140

Aparicio FM, Granger CWJ (1995) Nonlinear cointegration and some new tests for comovements modelling. Working Paper 95-15, Depart. of Economics of the University of California, San Diego

Aparicio FM, Escribano A, Garcia A (2006a) Synchronicity between financial time series: an exploratory analysis. Progress in Financial Markets Research, Nova Publishers (in press)

Aparicio FM, Escribano A, Garcia A (2006b) Range Unit Root (RUR) tests: robust against nonlinearities, error distributions, structural breaks and outliers. J Time Ser Anal 27:545-576

Arranz MA, Escribano A (2001) Cointegration testing under structural breaks: a robust extended error correction model. Oxf Bull Econ Stat 62:23-52

Balke NS, Fomby TB (1997) Threshold cointegration. International Economic Review, Department of Economics, University of Pennsylvania and Osaka University Institute of Social and Economic Research Association, vol 38(3), pp 627-645

Bec F, Rahbek A (2004) Vector equilibrium correction models with nonlinear discontinuous adjustments. Econom J 7:628-651

Bhattacharya P (1984) Induced-order statistics: theory and applications. Handbook of statistics. Elsevier, Amsterdam, vol 4, pp 383-403

Breitung J, Gouriéroux C (1997) Rank tests for unit roots. J Econom 81:7-27

Choi I, Saikkonen P (2004) Cointegrating smooth transition regressions. Econom Theory 20:301-340

Davidson J (1994) Stochastic limit theory. Oxford University Press, New York

Engle RF, Granger CWJ (1987) Cointegration and error correction: representation. Estimation and testing. Econometrica 55:251-276

Ericsson NR, Hendry DF, Prestwich KM (1997) The demand for broad money in the UK. International Finance Discussion Papers, 596

Escanciano JC, Escribano A (2007) Econometrics: nonlinear cointegration. Encyclopedia of complexity and systems science. Springer (forthcoming)

Escribano A (1986) Identification and modelling of economic relationships in a growing economy, Chap. IV. Ph.D. dissertation, University of California San Diego

Escribano A (1987a) Cointegration, time co-trends and error correction systems: an alternative approach. C.O.R.E. Discussion Paper, 8715, University of Louvain

Escribano A (1987b) Error correction systems: nonlinear adjustments to linear long-run relationships. C.O.R.E. Discussion Paper, 8730, University of Louvain

Escribano A (2004) Nonlinear error correction: the case of money demand in the UK (1878-2000). Macroecon Dyn 8:76-116

Escribano A, Mira S (2002) Nonlinear error correction models. J Time Ser Anal 23:509-522

Escribaro A, Sipols AE, Aparicio F (2006) Nonlinear cointegration and nonlinear error correction: applications of tests based on first differences of ranges. Commun Stat Simul Comput 35:939-956

Friedman M, Schwartz A (1982) Monetary trends in the USA and the UK: their relation to income, prices, and interest rates, 1867-1975. University of Chicago Press, Chicago

Granger CWJ (1981) Some properties of time series data and their use in econometric model specification. J Econom 16:121-130

Granger CWJ (1995) Modelling nonlinear relationships between extended memory variables. Econometrica 63(2):265-279

Granger CWJ, Hallman JJ (1991) Long-memory series with attractors. Oxf Bull Econ Stat 53:11-26 
Granger CWJ, Newbold P (1974) Spurious regressions in econometrics. J Econom 2:11-120

Granger CWJ, Teräsvirta T (1993) Modeling nonlinear economic relationships. Oxford University Press, New York

Hallman JJ (1990a) NonLinear integrated series, cointegration and an aplication. PhD Thesis, Department of Economics of the University of California at San Diego, La Jolla, USA

Hallman JJ (1990b) Cointegration and transformed series. Working Paper, 9014, Federal Reserve Bank of Cleveland

Hendry DF (1995) Dynamic econometrics. Oxford University Press, New York

Hendry DF, Ericsson NR (1991) An econometric analysis of UK money demand in monetary trends in the USA and UK by Milton Friedman and Anna J. Schwartz. Am Econ Rev 8

Horn RA, Johnson CR (1990) Matrix analysis. Schwartz. Cambridge Univ. Press, London, p 8

Johansen S (1988) Statistical analysis of cointegration vectors. J Econ Dyn Control 12:231-254

Johansen S (1991) Estimation and hypothesis testing of cointegrating vectors in Gaussian vector autoregressive models. Econometrica 59:1551-1580

Johansen S (1995) Likelihood-based inference in cointegrated vector auto-regressive models. Advanced texts in econometrics, Oxford University Press, New York

Kremers JJ, Ericsson NR, Dolado JJ (1992) The power of cointegration tests. Oxf Bull Econ Stat 54:325-348

Marmol F, Escribano A, Aparicio FM (2002) Instrumental variable interpretation of cointegration with inference results for fractional cointegration. Econom Theory 18:646-672

Park JY, Phillips PCB (2001) Nonlinear regressions with integrated time series. Econometrica 69:117-161

Phillips PCB (1987) Time series regression with a unit root. Econometrica 55:277-301

Saikkonen P (2005) Stability results for nonlinear error correction models. J Econom 127:69-81

Watson M (1994) Vector autoregressions and cointegration. Handbook Econom 4:2843-2945 